\title{
Enhancing the teaching of electromagnetics using differential forms
}

\author{
Richard H. Selfridge, Karl F. Warnick, and David V. Arnold \\ Brigham Young University
}

\section{Introduction:}

During the past three years we have introduced our electromagnetics students to differential forms. We teach a sequence of 3 courses in electromagnetic fundamentals at BYU. The sequence begins with basic principles and concludes with advanced concepts, including Greens functions, asymptotic methods, and anisotropic materials. In addition to these basic principles courses, we offer applications courses in antennas, microwave circuit design, remote sensing, radar, nonlinear and Fourier optics, and fiber optics. We have found that expressing basic electromagnetic principles in terms of differential forms as a supplement to vector analysis aids the students at all levels in understanding electromagnetic theory.

The use of differential forms is widespread in the physics community, particularly in gravitation and relativistic electrodynamics problems. Several researchers advocate the use of differential forms in electrical engineering, among the most outspoken is Burke.

The test of a mathematical formalism is shown in the applications. Although I have long been convinced of this, it was emphasized to me again when I decided to teach a graduate electrodynamics course using differential forms instead of the usual vector notation. I expected only modest gains, but in fact it made a tremendous improvement. The mathematics became "transparent" and the underlying physical structures became visible. (William L. Burke, Applied Differential Geometry, Cambridge University Press, 1985)

Proponents point out that forms provide additional insight into the nature of electromagnetics, simplify derivations, and provide notational compactness.

If differential forms are as beneficial as proponents claim, why have they not come into more widespread use in electrical engineering electromagnetics? One opinion is expressed by Georges A. Deschamps:

The differential forms approach has not yet had any impact on engineering in spite of its convenience, compactness, and many other qualities. The main reason for this is, of course, the lack of exposure in engineering publications: the entire literature on the subject of electromagnetics is written in vector calculus notation. It is hoped that this article will help remove this obstacle to a wider use of these techniques, and demonstrate some of the real advantages of this new notation. (Georges A. Deschamps, Fellow IEEE, Electromagnetics and Differential Forms, Proceedings of the IEEE, Vol. 69, No. 6, June 1981) 
Although Deschamps's article and others are quite useful in introducing differential forms to specialists in electromagnetics, they have done little to promote the use of differential forms in the broader electromagnetics community. If differential forms are to gain a wider acceptance in electrical engineering, not only should researchers and practitioners be made familiar with them, but students should be exposed to them in the undergraduate curriculum.

Presentation of differential forms to undergraduates in electrical engineering requires a different approach than teaching forms to graduates. In currently available articles and texts differential forms are usually presented in the most general and complex manner even to beginners. Presenters begin with differential geometry, exterior algebra, and complex metric space descriptions. This is contrary to the common approach used for teaching vector analysis. Vectors are presented from a simple algebraic and geometric point of view and complexity is added as needed. From our experiences, a successful introduction of differential forms to electrical engineering undergraduates begins with a simple presentation of forms as geometrical objects in a manner similar to the approach taken to introduce vectors. This paper outlines how we introduce differential forms to our undergraduate students. We show that even when the simplest problems in electromagnetics are explained using forms they yield valuable insights that are difficult to derive from the vector representation alone.

\section{A CONSTANT ELECTRIC FIELD}

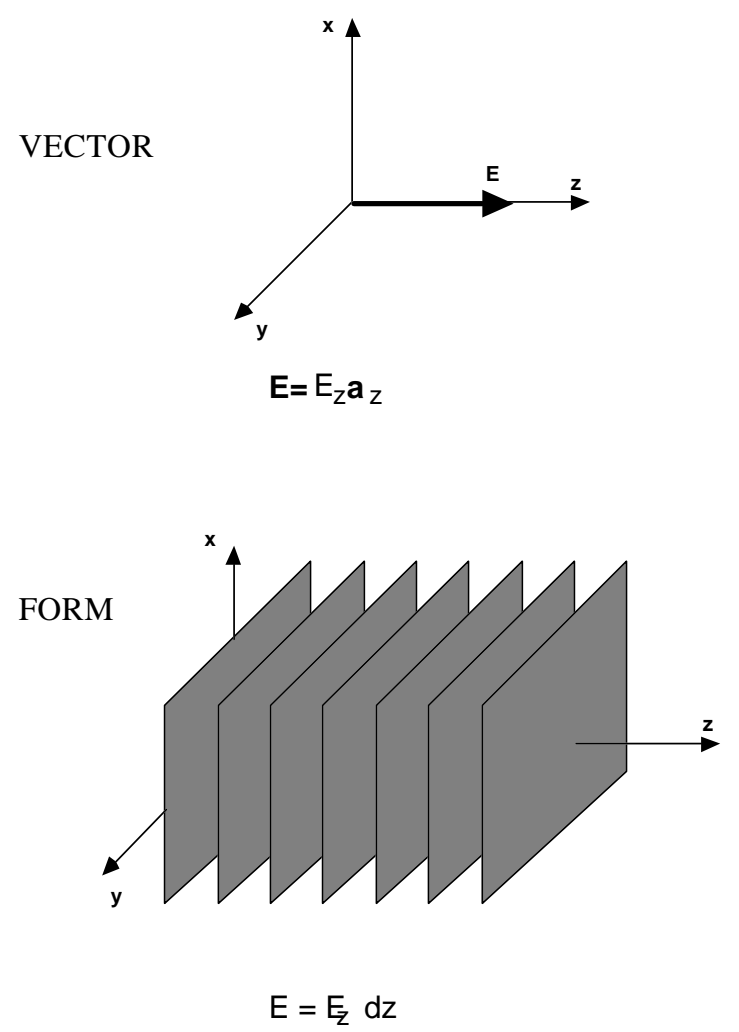

Figure 1. The vector and form representations of a constant electric field.

\section{A New Approach for Presenting Differential Forms}

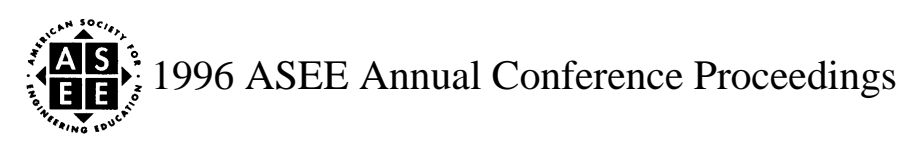


In our approach we show the differential form geometrically and algebraically without attempting to define all of its geometric and algebraic properties. Figure 1 shows an example of this more direct approach. It shows the vector and differential form representing the same constant E-field. The differential form associated with this constant electric field is represented as a series of plane surfaces of constant voltage perpendicular to the z-axis. The planes coincide with the equipotential surfaces. For constant field the surfaces are spaced equidistant from one another. Here the field vector is expressed as $E_{Z} \mathbf{a}_{\mathbf{Z}}$ Volts/meter. The corresponding algebraic representation in terms of forms is $E_{Z} d z$ Volts. The form associated with electric field is called a one form because it has only one differential multiplier. Notice that the dimensional units of the form representation are volts because it implies the multiplication of distance times the field.

Figure 2 shows the electric field vector and form representations of an E-field that increases in intensity away from the vertical axis. The spacing of the surfaces decreases with increasing field strength. In this case the electric field may be written as $z^{2} \mathbf{a}_{\mathbf{z}}$ and the form as $z^{2} \mathrm{dz}$. It is interesting to note that although we are used to representing increasing field strength with a higher density of field lines, it is not in keeping with the definition of vectors. The length, not the spacing, of a vector determines its relative magnitude in the fundamental definition of vectors.

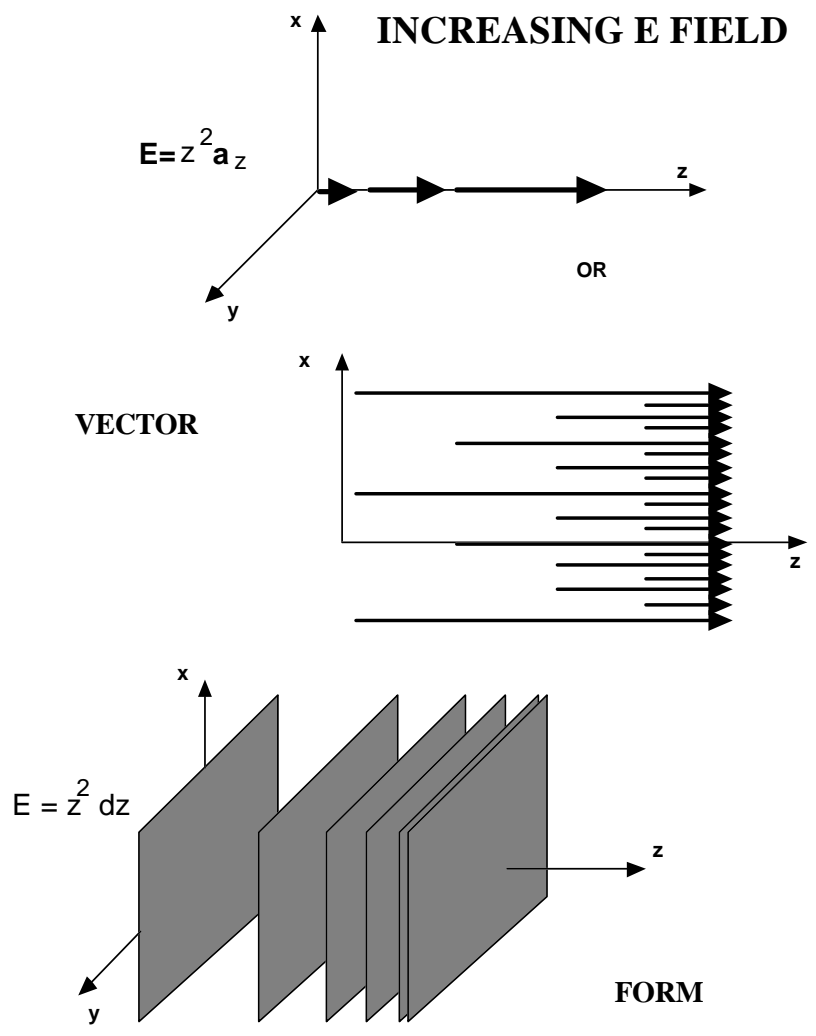

Figure 2. Forms and vectors for an increasing E-field.

We introduce the electric flux density as an important example of a two form. It is convenient to illustrate electric flux using the parallel plate capacitor. 


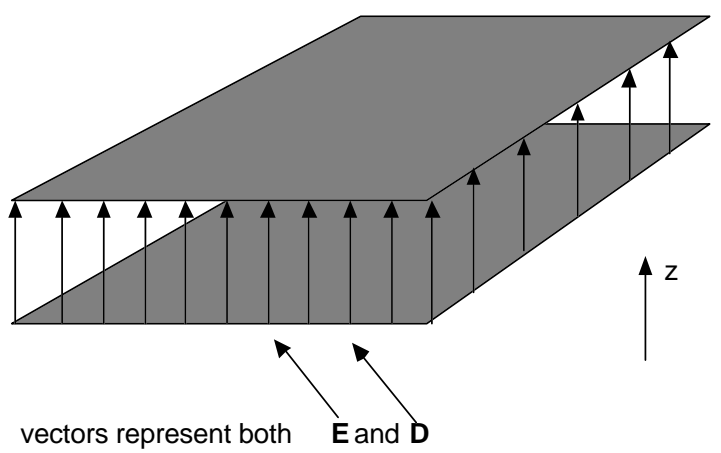

Figure 3. Electric field and flux density vectors for a parallel plate capacitor.

Upon application of a voltage difference between the two plates an electric field is created in the region between the two plates. The vector and forms for this field are shown in Figures 3 and 4, respectively. The flux density is found from the E-field by applying the constitutive relationship between the electric field and its associated flux density. For vectors (in homogeneous isotropic media) this is written as $\mathbf{D}=\varepsilon \mathbf{E}$, where $\varepsilon$ is a scalar quantity. From the figure we see that besides the scaling there is no apparent difference between flux and field in the vector description.

The constitutive relation in forms is written as $\mathrm{D}=\varepsilon^{*} \mathrm{E}$ where, ${ }^{*}$, is the hodge star operator. In a three dimensional Cartesian space it transforms the one form $\mathrm{dx}$ to the two form dydz, dy to dzdx, and dz to dxdy. In the case shown, the operation $\varepsilon^{*} E_{Z} d z$, produces a flux $D=\varepsilon E_{Z} d x d y$. The dimensional units of the flux form are Coulombs. Figure 4 shows that $\mathrm{dx}$ creates a series of planes perpendicular to the $\mathrm{x}$-axis and dy is a series of planes perpendicular to the y-axis.

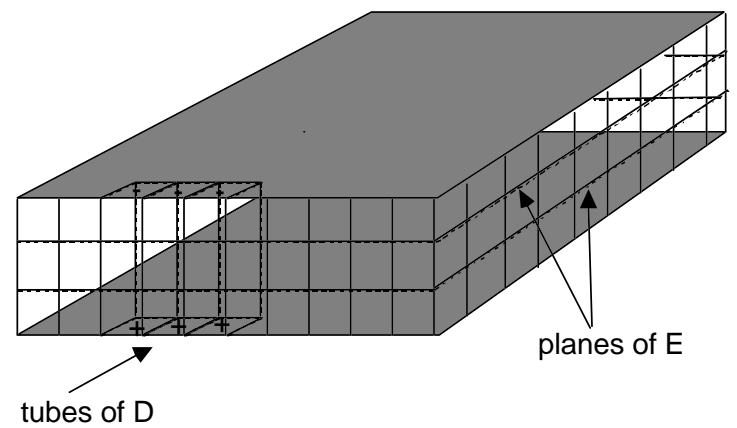

Figure 4. Forms show clearly the difference between flux and field quantities.

The combination generates a group of tubes opening in the z-direction. There is a clear distinction between the electric field and electric flux. The field form is a series of planes perpendicular to the plates and the flux form is an array of tubes joining the positive charge on the bottom plate with the negative charge on the top plate. The spatial density of the tubes indicates the magnitude of the flux, more tubes indicate a higher flux density. In our experience in teaching electromagnetics the use of forms in this simple problem clearly illuminates the difference between fields and fluxes. In vector notation the field and flux are both vectors. In forms the field is a one form and the flux is a two form.

We have seen that the electric field and magnetic field are conveniently expressed as one forms and that electric and magnetic fluxes are two forms. Combining a one form and a two form gives a three form as shown in Figure 5. The combination of the electric field and flux to arrive at the energy density in vector 
notation is a dot product, $\mathrm{w}=\mathbf{D} \bullet \mathbf{E}$. In forms we use the hook or exterior product, $\mathrm{w}=\mathrm{D} \wedge \mathrm{E}$. The hook product shows the resulting energy density is a three form. The geometric representation of the three form is the cube formed by the combination of the three planes associated with the one forms $\mathrm{dx}, \mathrm{dy}$, and $\mathrm{dz}$.

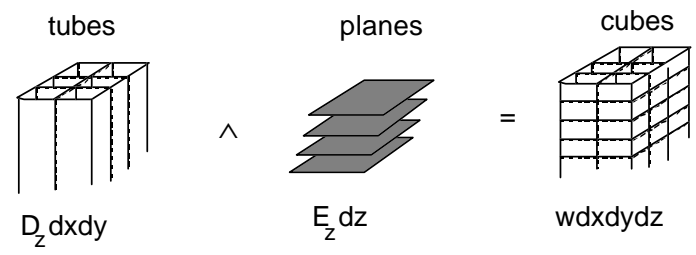

Figure 5. The exterior product of a two form and a one form yields a three form.

We also use forms to make a clear distinction between magnetic flux and field. The air gap represented in the magnet shown in Figure 6 demonstrates this difference. The magnetic field, H, is depicted as a series of planes and the magnetic flux is shown using the tubes. Here the relationship between the two quantities is $\mathrm{B}=\mu^{*} \mathrm{H}$.

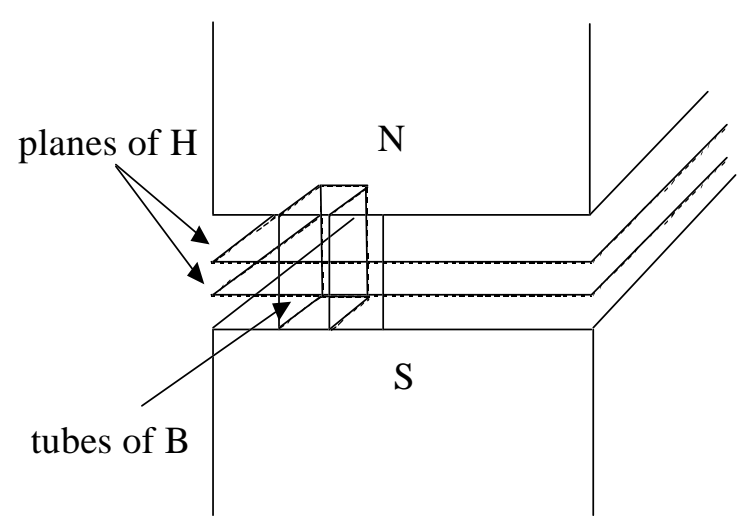

\section{Figure 6. The magnetic field and flux forms in the air gap between two poles of a magnet.}

One commonly expressed definition of forms is that they are the things under integral signs. The integration of forms is very natural because the differential element of integration is carried in the form. The integral for a one form is a line integral. This same ease of integration extends to two forms and three forms. Two forms are integrated over surfaces and three forms are amenable to volume integrals.

Although the examples we have shown to this point have been in Cartesian coordinates, differential forms are also useful in other curvilinear coordinate systems. Figure 7 shows a cross section of the field and flux associated with a point charge. It shows that the vector field and flux both are represented with vectors pointing away from the origin. Using forms the electric field is a series of concentric circles and the flux tubes are shown opening away from the charge. Again, the difference between flux and field is clear. 


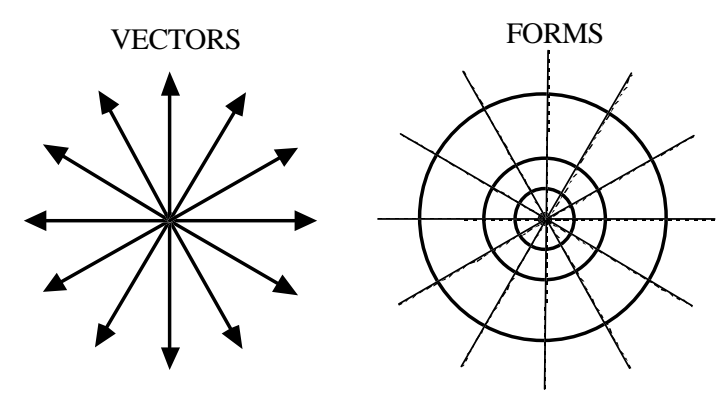

Figure 7. Cross section of the E-field and D-field associated with a point charge.

Maxwell's equations are more simply expressed in terms of differential forms than in vectors. In forms we write:

$$
\begin{aligned}
& d D=\rho \\
& d B=0 \\
& d E=-\frac{\partial B}{\partial t} \\
& d H=\frac{\partial D}{\partial t}+J
\end{aligned}
$$

In these expressions there is an implied exterior product between the form and the $d$ operator. The $d$ operator is similar to the $\nabla$ operator in vectors. In Cartesian coordinates it is expressed as:

$$
d=\left(\frac{\partial}{\partial x} d x+\frac{\partial}{\partial y} d y+\frac{\partial}{\partial z} d z\right)
$$

The exterior product of the one form differential operator on another one form creates a two form, but it generates a three form when it is wedge multiplied by a two form. In the equation representing gauss's law we can see that the one form differential operator converts the two form flux into a three form cube.

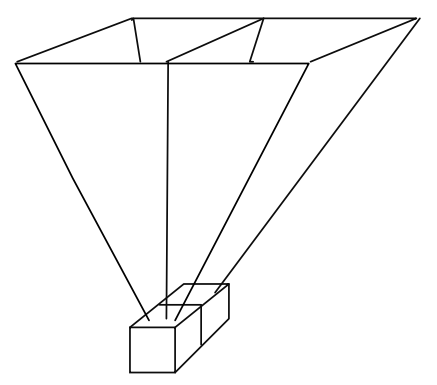

Figure 8. Geometric description of gauss's law using differential forms.

From the differential forms representation of Maxwell's equations we see a clear algebraic similarity between gauss's law and ampere's law. The one form differential operator applied to the one form E-field produces a two form flux or electric current density. This is also manifest in the geometry of the forms. Gauss's law is shown in Figure 8 where tubes of flux emanate from cubes of charge. Similarly amperes law is manifest in Figure 9 where E-form planes are generated by tubes of current or time changing electric flux. 


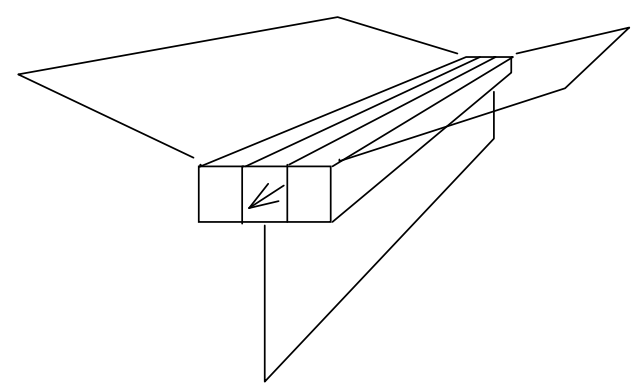

Figure 9. Geometric description of ampere's law using differential forms.

\section{Conclusion}

We have seen that students enjoy learning about differential forms and applying them to electromagnetics problems. More significantly they gain a more thorough understanding of the basic principles of electromagnetic fields when they are introduced to the concepts of differential forms early in their study of electromagnetics. In addition, if the same model holds true for electrical engineering as for physics, the application of differential forms to certain classes of problems may allow us to solve new problems that are all but impossible using vectors.

Using differential forms as a supplement to vectors broadens the vocabulary and images available to the teacher in presenting electromagnetics. From the professors' point of view, we have found that once we have used differential forms to teach about electromagnetics if we attempt to restrict ourselves to teaching with the use of vectors only, it is very frustrating, as if our hands were tied behind our backs. We hope many of you will incorporate forms into your teaching and find the same success and enjoyment we have.

\section{Biographies}

-DICK SELFRIDGE received his Ph.D. in electrical engineering from the University of California at Davis. His research interests are in optical fiber devices, recursive greens functions, and electromagnetics. $\mathrm{He}$ is currently an associate professor at Brigham Young University in the Department of Electrical and Computer Engineering.

-KARL WARNICK is a Ph.D. candidate in Electrical Engineering at Brigham Young University. His is a National Science Foundation Graduate Fellow. Current interests include topology, differential forms and other aspects of differential geometry and electromagnetic field theory.

-DAVID ARNOLD received BS and MS degrees from Brigham Young University, and a Ph.D. from Massachusetts Institute of Technology. His research interests are in electromagnetic theory and microwave remote sensing. He is currently an assistant professor in the Electrical and Computer Engineering Department at Brigham Young University. 